\title{
2HOT: An Improved Parallel Hashed Oct-Tree N-Body Algorithm for Cosmological Simulation
}

\author{
Michael S. Warren \\ Theoretical Division \\ Los Alamos National Laboratory \\ msw@lanl.gov
}

\begin{abstract}
We report on improvements made over the past two decades to our adaptive treecode N-body method (HOT). A mathematical and computational approach to the cosmological N-body problem is described, with performance and scalability measured up to $256 \mathrm{k}\left(2^{18}\right)$ processors. We present error analysis and scientific application results from a series of more than ten 69 billion $\left(4096^{3}\right)$ particle cosmological simulations, accounting for $4 \times 10^{20}$ floating point operations. These results include the first simulations using the new constraints on the standard model of cosmology from the Planck satellite. Our simulations set a new standard for accuracy and scientific throughput, while meeting or exceeding the computational efficiency of the latest generation of hybrid TreePM N-body methods.
\end{abstract}

\section{General Terms}

Computational Cosmology, N-body, Fast Multipole Method

\section{INTRODUCTION}

We first reported on our parallel N-body algorithm (HOT) 20 years ago 67] (hereafter WS93). Over the same timescale, cosmology has been transformed from a qualitative to a quantitative science. Constrained by a diverse suite of observations [47, 49, 53, 44, 9, the parameters describing the large-scale Universe are now known to near $1 \%$ precision. In this paper, we describe an improved version of our code (2HOT), and present a suite of simulations which probe the finest details of our current understanding of cosmology.

Computer simulations enable discovery. In the words of the Astronomy and Astrophysics Decadal Survey, "Through computer modeling, we understand the deep implications of our very detailed observational data and formulate new theories to stimulate further observations" [11. The only way to accurately model the evolution of dark matter in the Universe is through the use of advanced algorithms on massively parallel computers (see 29] for a recent review). The origin of cosmic structure and the global evolution of the Universe

Permission to make digital or hard copies of part or all of this work for personal or classroom use is granted without fee provided that copies are not made or distributed for profit or commercial advantage and that copies bear this notice and the full citation on the first page. Copyrights for thirdparty components of this work must be honored. For all other uses, contact the Owner/Author.

Copyright is held by the owner/author(s).

SC '13 Nov 17-21 2013, Denver, CO, USA

ACM 978-1-4503-2378-9/13/11.

http://dx.doi.org/10.1145/2503210.2503220 can be probed by selecting a set of cosmological parameters, modeling the growth of structure, and then comparing the model to the observations (Figure 1).
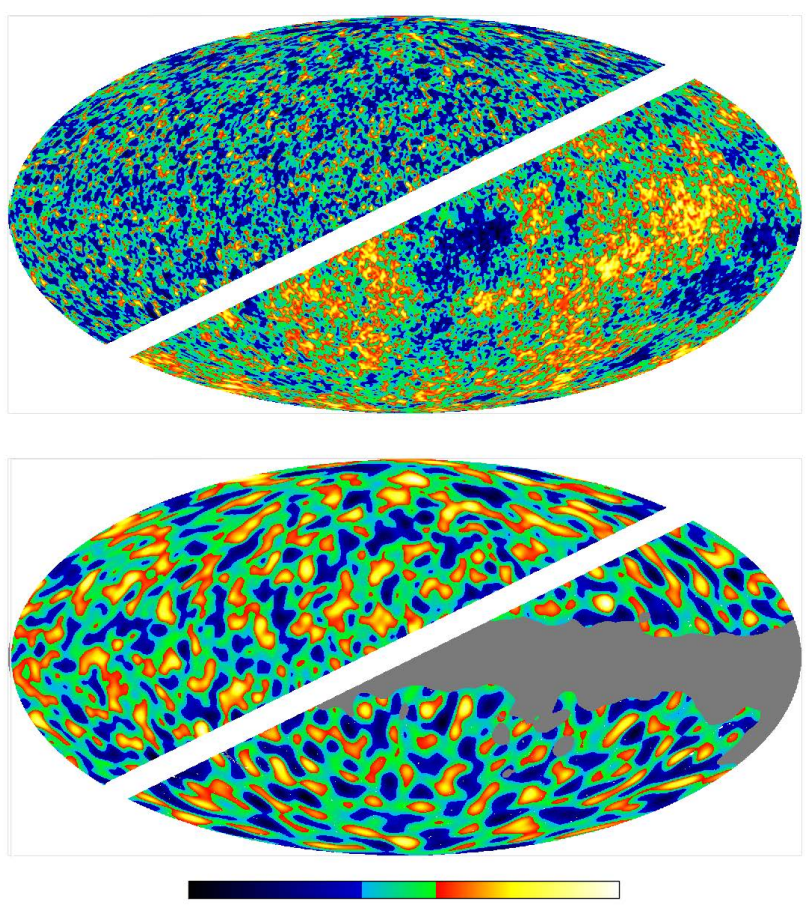

Figure 1: Recent results from the Planck satellite [9] compared with light-cone output from 2HOT. We present our numerical simulation results in the same HEALPix $^{1}$ [21] Mollewide projection of the celestial sphere used by Planck. The upper figure shows the density of dark matter in a 69 billion particle simulation (upper left) compared with the fluctuations in the cosmic microwave background. The obvious difference in the upper panel is due to the imperfect removal of sources within our galaxy in the Planck data, The statistical measurements of the smaller details match precisely between the observation and simulation. The lower figure shows the simulation compared with the gravitational lensing signal measured by Planck.

\footnotetext{
$\sqrt[1]{\text { http://healpix.jpl.nasa.gov }}$
} 
Computer simulations are playing an increasingly important role in the modern scientific method, yet the exponential pace of growth in the size of calculations does not necessarily translate into better tests of our scientific models or increased understanding of our Universe. Anywhere the relatively slow growth in the capacity of human attention intersects with the exponential explosion of information, new tensions are created. The timespan between the completion of a large simulation and the publication of scientific results based upon it is now often a year or more, and is growing longer instead of shorter. In the application described here, the sheer complexity of managing the volume of information in many layers of data and code has required additional software tools to be developed. We have written substantially more lines of software for data analysis, generating initial conditions, testing and task management than are present in the $2 \mathrm{HOT}$ code base. The scale of simulations requires most of these ancillary tools to be parallel as well.

High-performance computing (HPC) allows us to probe more questions with increased resolution and reduced statistical uncertainty, leading to new scientific discoveries. However, reducing the statistical errors more often than not uncovers systematic errors previously masked by statistical variance. Addressing these details takes us out of realm of HPC into applied mathematics, software engineering and data analysis. However, without progress on all fronts, the over-arching scientific questions can not be answered. A corollary of this point is that making a code faster is often a poor investment when the aim is to answer a particular scientific question. More important than speed is the code's applicability to the problem, correctness, and even less tangible properties such as robustness and maintainability. For those reasons, we focus here on the wide variety of changes made to $2 \mathrm{HOT}$ over the past two decades which have enabled us to produce the state-of-the-art scientific results presented in Section 6 .

One of our first scientific N-body simulations of dark matter [70] used 1.1 million particles and was performed on the 64-node Caltech/JPL Mark III hypercube in 1990. The simulation was completed in 60 hours, sustaining $160 \mathrm{Mflop} / \mathrm{s}$ with a parallel efficiency of $85 \%$. In 2012 we used 2 HOT on 262 thousand processors with over one trillion $\left(10^{12}\right)$ particles, sustaining in excess of 1.6 Petaflops with a parallel efficiency of $90 \%$ 63. Since our first parallel treecode simulations, the message-passing programming model, time to solution and parallel efficiency are nearly the same, but the problem size has increased by a factor of a million, and performance a factor of 10 million.

Since WS93, HOT was been extended and optimized to be applicable to more general problems such as incompressible fluid flow with the vortex particle method 41. and astrophysical gas dynamics with smoothed particle hydrodynamics [19, 18, 15. The code also won the Gordon Bell performance prize and price/performance prize in 1997 60. and 1998 65. It was an early driver of Linux-based cluster architectures 60, 62, 64 and helped call attention to power issues 69, 16]. Perhaps surprisingly (given that WS93 was presented at the same conference as the draft MPI 1.0 standard), the fundamental HPC abstractions in the code have changed little over two decades, while more significant changes have been required in its mathematical and cosmological underpinnings.

\section{MATHEMATICAL APPROACH}

\subsection{Equations of Motion}

The mathematical equations governing the evolution of structure in an expanding Universe are generally solved using comoving coordinates, $\vec{x}=\vec{r} / a(t) . \vec{r}$ is the "proper" coordinate, while the scale factor $a(t)$ is defined via the Friedmann equation

$$
\left(H / H_{0}\right)^{2}=\Omega_{R} / a^{4}+\Omega_{M} / a^{3}+\Omega_{k} / a^{2}+\Omega_{D E}
$$

in terms of the Hubble parameter $H \equiv \dot{a} / a$ and the densities of the various components of the Universe; radiation in the form of photons and ultra-relativistic particles $\left(\Omega_{R}\right)$, mass in the form of cold dark matter and baryons $\left(\Omega_{M}\right)$, spatial curvature $\left(\Omega_{k}\right)$ and dark energy or a cosmological constant $\left(\Omega_{D E}\right)$. The particle dynamics are defined in terms of the motion relative to the background model, the scale factor and the acceleration due to gravity [39],

$$
\frac{d \vec{v}_{i}}{d t}+2 \frac{\dot{a}}{a} \overrightarrow{v_{i}}=-\frac{1}{a^{3}} \sum_{i \neq j} \frac{G m_{j} \vec{x}_{i j}}{\left|x_{i j}\right|^{3}}
$$

Cosmological evolution codes most often account for cold dark matter, baryons and dark energy. The Boltzmann solvers which calculate the power spectrum of density perturbations use all of the components, including photons and massless and massive neutrinos. For precise computations, it is now necessary to include these other species. Using the parameters of the Planck 2013 cosmological model, the age of the Universe is 3.7 million years older if photons and radiation from massless neutrinos are not treated correctly. The linear growth factor from redshift 99 (an expansion of 100) changes by almost $5 \%$ (from 82.8 to 79.0 ) under the same circumstances. $2 \mathrm{HOT}$ integrates directly with the computation of the background quantities and growth function provided by CLASS 30, either in tabular form or by linking directly with the CLASS library, and thereby supports any cosmology which can be defined in CLASS. 2HOT additionally maintains the ability to calculate the scale factor and linear growth factor analytically (when radiation or nontrivial dark energy is not included) in order to be able to directly compare with codes which do not yet support them.

\subsection{Multipole Methods}

Using $N$ particles to represent the Universe, treecodes and fast multipole methods reduce the $N^{2}$ scaling of the righthand side of equation (2) to $O(N)$ or $O(N \log N)$ - a significant savings for current cosmological simulations which use $N$ in the range of $10^{10}$ to $10^{12}$.

\subsubsection{Background Subtraction}

Large cosmological simulations present a unique set of challenges for multipole methods. The Universe is nearly uniform at large scales. This means the resultant acceleration on a particle from distant regions is a sum of large terms which mostly cancel. We can precisely quantify this effect by looking at the variance of density in spheres of radius $r$, which is an integral of the power spectrum convolved with a top-hat window,

$$
\int_{0}^{\infty}(d k / k) \delta_{k}^{2} W(k r)^{2}
$$

For a sphere of radius $100 \mathrm{Mpc} / \mathrm{h}$, the variance is 0.068 of the mean value for the standard model. This value scales 


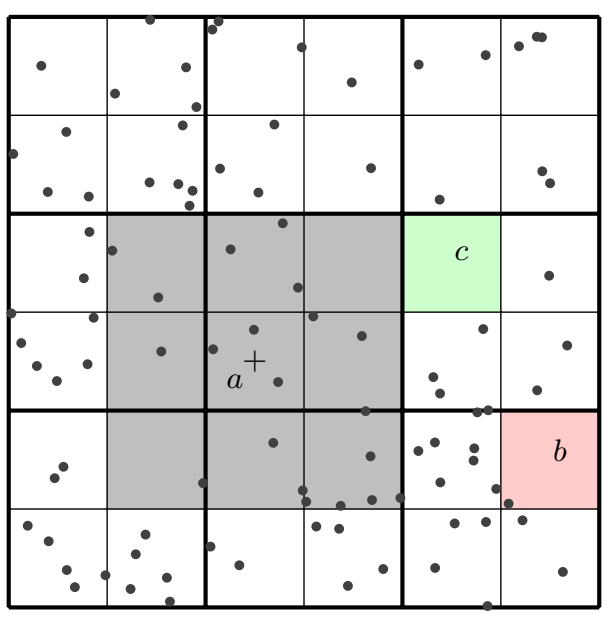

Figure 2: An illustration of background subtraction, which greatly improves the performance of the treecode algorithm for nearly uniform mass distributions (such as large-volume cosmological simulations, especially at early times). The bodies inside cell $a$ interact with the bodies and cells inside the gray shaded area as usual. Bodies inside cell $a$ interact with all other cells ( $b$, for example) after the background contribution of a uniform density cube is subtracted from the multipole expansion. Empty cell $c$ (which would be ignored in the usual algorithm) must have its background contribution subtracted as well. The background contribution of the gray shaded area to the calculated force and potential of the bodies in $a$ is removed analytically.

with the growth of cosmic structure over time, so at the beginning of a simulation it will be a factor of 50-100 lower. At early times when we calculate the acceleration from a 100 Mpc cell in one direction, $99 \%$ of that value will cancel with a cell in the opposite direction, leaving a small remainder (the "peculiar" acceleration). This implies that the error tolerance needed for these large cells is 100 times stricter than for the short-range interactions. For larger volumes or earlier starting times, even more accuracy is required. This suggests that eliminating the background contribution from the partial acceleration terms would be beneficial.

The mathematical equations describing the evolving Universe subtract the uniform background, accounting for it in the evolution of the scale factor $a(t)$. Fourier-based codes do this automatically, since the DC component has no dynamical effect. For treecodes, the proper approach is less obvious. Essentially, we wish to convert the always-positive mass distribution into density perturbations $\delta \rho / \rho$. These density contrasts can be positive or negative, making the gravitational problem analogous to an electrostatics problem, with positive and negative charges.

Since we wish to retain the particle-based representation of the density, the background subtraction can be obtained by adding the multipole expansion of a cube of uniform negative density to each interaction. Since the multipole expansion of a cube is fairly simple due to symmetries, this can be done with a few operations if the multipole expansions are with respect to the cell centers (rather than the center of mass). This in turn adds a few operations to the interaction routines, since dipole moments are now present. At scales near the inter-particle separation, this approach breaks down, since any empty cells which would be ignored in a direct summation must be accounted for, as well as requiring high-order expansions for neighboring cells with only a few particles, which would normally be calculated with cheaper monopole interactions. Rather than modify each interaction for the near field, we define a larger cube which approximately surrounds the local region of empty and single particle cells and calculate the background acceleration within the surrounding cell (Figure 2). This acceleration term can be done with a multipole and local expansion, or our current approach of using the analytic expression for the force inside a uniform cube [59, 46].

A subtle point is that in the far-field we only want to subtract the uniform background expansion up to the same order as the multipole expansion of the matter to minimize the error. If a cube of particles is expanded to order $p=4$, the $p=6$ and higher multipoles from the background are not included, so they should not be subtracted. Using background subtraction increases the cost of each interaction somewhat, but results in a huge improvement in overall efficiency, since many fewer interactions need to be computed. At early times we have measured an improvement of a factor of five. The multipole acceptance criterion (MAC) based on an absolute error also becomes much better behaved, leading to improved error behavior as well.

\subsubsection{Multipole Error Bounds}

A critical ingredient of any optimized multipole method is the mathematical machinery to bound or estimate the error in the interactions. The methods we previously developed [45, 68, allow us to dynamically decide between using different orders of expansion or refinement, automatically choosing the most efficient method to achieve a given accuracy.

The expressions we derived in 68] support methods which use both multipole and local expansions (cell-cell interactions) and those which use only multipole expansions (cellbody interactions with $\Delta=0$ ). The scaling of these methods with $N$ depends on precisely how the error is constrained while increasing $N$, but generally methods which support cell-cell interactions scale as $O(N)$ and those that do not scale as $O(N \log N)$. Our experience has been that using $O(N)$-type algorithms for cosmological simulation exposes some undesirable behaviors. In particular, the behavior of the errors near the outer regions of local expansions are highly correlated. To suppress the accumulation of these errors, the accuracy of the local expansion must be increased, or their spatial scale reduced to the point where the benefit of the $O(N)$ method is questionable, at least at the modest accuracies of current cosmological simulations. For this reason, we have focused on the implementation and optimization of an $O(N \log N)$ method.

Consider a configuration of sources as in Figure 3 The sources are contained within a "source" cell, $\mathcal{V}$ of radius $b_{\max }$, while the field is evaluated at separation $B_{(\Delta)}$ from $B_{(x)_{0}}$, the center of "sink" cell $\mathcal{W}$.

In terms of an arbitrary Green's function, $G$, the field is:

$$
\phi\left(B_{(x)}\right)=\int_{\mathcal{V}} d B_{(y)} G\left(B_{(x)}-B_{(y)}\right) \rho\left(B_{(y)}\right)
$$

Expanding $\mathrm{G}$ around $B_{(R)_{0}}=B_{(x)_{0}}-B_{(y)_{0}}$ in a Taylor 
series leads to the Cartesian multipole expansion:

$$
\begin{array}{r}
\phi\left(B_{(x)}\right)=\sum_{n=0}^{p} \frac{(-1)^{n}}{n !} \partial_{(n)} G\left(B_{(R)_{0}}\right) \odot B_{(M)}{ }^{(n)} \\
\left(B_{(y)_{0}}+B_{(\Delta)}\right)+\Phi_{(p)}\left(B_{(x)}\right)
\end{array}
$$

where $\Phi_{(p)}$ is the error term, and the moment tensor is defined relative to a center, $B_{(z)}$ as:

$$
M^{(n)}\left(B_{(z)}\right)=\int d B_{(y)}\left(B_{(y)}-B_{(z)}\right)^{(n)} \rho\left(B_{(y)}\right)
$$

We have used a notational shorthand in which $B_{(v)}^{(n)}$ indicates the n-fold outer product of the vector $B_{(v)}$ with itself, while $\odot$ indicates a tensor inner-product and $\partial_{(n)} G$ indicates the rank- $n$ tensor whose components are the partial derivatives of $\mathrm{G}$ in the Cartesian directions. We can further expand the result by writing $B_{(M)}{ }^{(n)}\left(B_{(y)_{0}}+B_{(\Delta)}\right)$ as a sum over powers of the components of $B_{(\Delta)}$, and then recollecting terms (see Eqns $12-14$ in 68]).

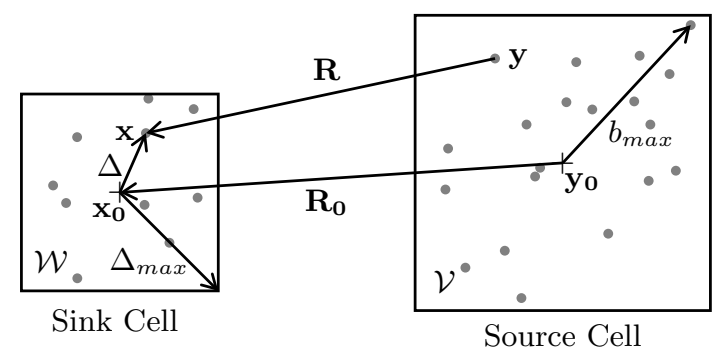

Figure 3: An illustration of the relevant distances used in the multipole expansion and error bound equations.

While the mathematical notation above is compact, translating this representation to an optimized interaction routine is non-trivial. The expression for the force with $p=8$ in three dimensions begins with $3^{8}=6561$ terms. We resort to metaprogramming, translating the intermediate representation of the computer algebra system 74 directly into $\mathrm{C}$ code. This approach is capable of producing the necessary interaction routines through $p=8$ without human intervention. A better approach would combine a compiler with knowledge of the computing architecture into the symbolic algebra system, allowing very high-level optimizations using mathematical equivalences that are lost once the formulae are expressed in a general programming language. To our knowledge, no such system currently exists.

We have also investigated support for pseudo-particle 28 . and kernel-independent [76] approaches which abstract the multipole interactions to more easily computed equations. For instance, the pseudo-particle method allows one to represent the far field of many particles as a set of pseudoparticle monopole interactions. We have found that such approaches are not as efficient as a well-coded multipole interaction routine in the case of gravitational or Coulombic interactions, at least up to order $p=8$.

\subsection{Time Integration}

The original version of HOT integrated the equations of motion using the leapfrog techniques described in [14], with a logarithmic timestep at early times. This approach has proven inadequate for high-accuracy simulations. Fortunately, the theory for symplectic time integration in a comoving background was developed by 42, which we have fully adopted. The advantages of this integrator are discussed in detail in [50]. We calculate the necessary integrals for the "drift" and "kick" operators in arbitrary cosmologies with code added to the background calculations in CLASS 30. We additionally restrict the changes of the timestep to exact factors of two, rather than allowing incremental changes at early times. Any change of timestep breaks the symplectic property of the integrator, but making occasional larger adjustments rather than continuous small adjustment (as is done in GADGET2 [50]) appears to provide slightly better convergence properties. We have also modified $2 \mathrm{HOT}$ to save "checkpoint" files which maintain the leapfrog offset between position and velocity. This allows the code to maintain 2nd-order accuracy in the time integration when restarting from a saved file. Otherwise, the initial (first order) step in the leapfrog scheme can lead to detectable errors after restarting at early times.

\subsection{Boundary Conditions}

Periodic boundary conditions have been applied to multipole methods in a variety of ways, but most often are variants of the Ewald method [25]. For 2HOT, we have adopted the approach described in [8, which is based on the central result of Nijboer \& De Wette (1957) 36]. Effectively the same method in a Cartesian basis was first used in a cosmological simulation by Metchnik 35. This method sums the infinite series of each relevant combination of powers of the co-ordinates, which can be taken outside the sum of periodic replicas (since the multipole expansion of each replica is identical). These pre-computed coefficients are then used in a local expansion about the center of the volume. We use $p=8$ and $w s=2$, which accounts for the boundary effects to near single-precision floating point accuracy (one part in $10^{-7}$ ). The computational expense of this approach is about $1 \%$ of the total force calculation for the local expansion, and $5-10 \%$ for the 124 boundary cubes, depending on the overall accuracy tolerance.

\subsection{Force Smoothing}

The standard practice in cosmological N-body simulations is to smooth the forces at small scales, usually with a Plummer or spline 50 kernel. We have implemented these smoothing kernels in 2HOT, as well as the additional kernels described by Dehnen 13. Dehnen concludes that the optimal softening method uses a compensating kernel, with forces that are higher than the Newtonian force at the outer edge of the smoothing kernel, which compensates for the lower forces in the interior and serves to reduce the bias in the force calculation. Our tests confirm these conclusions, and we use Dehnen's $K 1$ compensating kernel for our simulations, except for the tests comparing directly to other codes.

\section{COMPUTATIONAL APPROACH}

\subsection{Domain Decomposition}

The space-filling curve domain decomposition approach we proposed in WS93 has been widely adopted in both application codes (e.g. [23, 50, 27, 75]) and more general libraries 38, 32. Our claim that such orderings are also beneficial for improving memory hierarchy performance has 
also been validated [50, 34]. We show an example of a 3-d decomposition of 3072 processor domains in Figure 4

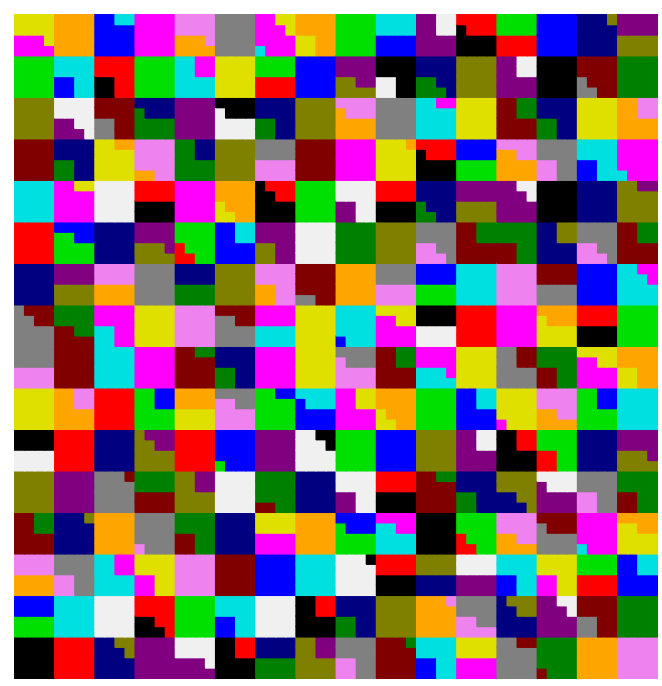

Figure 4: A demonstration of our space-filling curve domain decomposition for a highly evolved cosmological simulation on 3072 processors in a cube 1 $\mathrm{Gpc} / \mathrm{h}$ across. We view one face of the 3 -d computational volume, cycling through 16 different colors in turn for each processor domain. Starting in the lower left, the sequence goes black, red, green, blue, cyan, and then continues underneath in the $z$ dimension (not visible), returning to the front face with dark blue, brown, purple, white, etc.

The mapping of spatial co-ordinates to integer keys described in WS93 converts the domain decomposition problem into a generalized parallel sort. The method we use is similar to the sample sort described in 48, with the onnode portion done with an American flag radix sort 33. After using the samples to determine the edges of the processor domains, in the initial HOT implementation the data was moved using a loop over all pairs of processors needing to exchange data. We converted the data exchange to use MPI_Alltoall() for improved scalability. This exposed problems in the implementation of Alltoall on large machines for both OpenMPI and the Cray system MPI. The first "scalability surprise" was related to the way buffers were managed internally in OpenMPI, with the number of communication buffers scaling as the number of processes squared. This did not allow our code to run on more than 256 24-core nodes using OpenMPI. We had to rewrite the implementation of Alltoall using a hierarchical approach, with only one process per node relaying messages to other nodes. The second was a "performance surprise" as defined by [54, where replacing the Cray system implementation of MPI_Alltoall() with a trivial implementation using a loop over all pairs of processes exchanging data led to a huge performance improvement when using more than $32 \mathrm{k}$ processors. Note that after the initial decomposition, the Alltoall communication pat- tern is very sparse, since particles will only move to a small number of neighboring domains during a timestep. This also allows significant optimization of the sample sort, since the samples can be well-placed with respect to the splits in the previous decomposition.

\subsection{Tree Construction and Traversal}

The parallel tree construction in WS93 used a global concatenation of a set of "branch" nodes from each processor to construct the tree at levels coarser than the individual processor domains. While this is an adequate solution up to a few thousand processors, at the level of tens of thousands of domains and larger, it leads to unacceptable overhead. Most of the nodes communicated and stored will never be used directly, since the local traversal will only probe that deeply in the tree near its own spatial domain. Instead of a global concatenation, we proceed with a pairwise hierarchical aggregation loop over $i$ up to $\log _{2} N_{\text {proc }}$ by exchanging branch nodes between nearest neighbors in the 1-d space-filling curve, incrementally updating the tree with those nodes, then doing the same with the $2^{i}$-th neighbor. This provides a minimal set of shared upper-level nodes for each processor domain, and has demonstrated its scalability to $256 \mathrm{k}$ processors.

In 68. we describe a tree traversal abstraction which enables a variety of interactions to be expressed between "source" and "sink" nodes in tree data structures. This abstraction has since been termed dual-tree traversal [22, 77. The dualtree traversal is a key component of our method to increase the instruction-level parallelism in the code to better enable new CPU and GPU architectures (see Section 3.3.).

During the tree traversal we use the same request/reply protocol described in WS93 using the global key labels assigned during the tree construction phase. Additional bits to label the source processor have been added to the hcells to support machines with up to $2^{18}$ processors. Our initial approach to hiding latency in the tree traversal was recast in the form of an active message abstraction. We believe that such event-driven handlers are more robust and less errorprone to implement correctly 37. We currently use our own implementation of active messages within MPI, which we call "Asynchronous Batched Messages" (ABM). ABM is a key component of our ability to overlap communication and computation and hide message latency. MPI has supported one-sided communications primitives for many years, but their performance is often worse than regular point-topoint communication. It is likely that synchronization and locking overheads and complexity are to blame 3. Newer implementations of active messages 73 are an attractive alternative, which we plan to implement as time allows.

\subsection{Improving instruction-level parallelism}

In WS93 we used the fact that particles which are spatially near each other tend to have very similar cell interaction lists. By updating the particles in an order which takes advantage of their spatial proximity, we improved the performance of the memory hierarchy. Going beyond this optimization with dual-tree traversal, we can bundle a set of $m$ source cells which have interactions in common with a set of $n$ sink particles (contained within a sink cell), and perform the full $m \times n$ interactions on this block. This further improves cache behavior on CPU architectures, and enables a simple way for GPU co-processors to provide reasonable speedup, even in the face of limited peripheral bus band- 
width. We can further perform data reorganization on the source cells (such as swizzling from an array-of-structures to a structure-of-arrays for SIMD processors) to improve performance, and have this cost shared among the $n$ sinks. In an $m \times n$ interaction scheme, the interaction vector for a single sink is computed in several stages, which requires writing the intermediate results back to memory multiple times, in contrast to the WS93 method which required only one write per sink. For current architectures, the write bandwidth available is easily sufficient to support the $m \times n$ blocking.

Taking advantage of instruction-level parallelism is essential. In the past, obtaining good CPU performance for gravitational kernels often required hand-tuned assembly code. Implementing the complex high-order multipole interactions using assembly code would be extremely difficult. Fortunately, the gcc compiler comes to the rescue with vector intrinsics 51. We use gcc's vector_size attribute, which directs the compiler to use SSE or AVX vector instructions for the labeled variables. By providing the interaction functions with the appropriately aligned and interleaved data, gcc is able to obtain near optimal SIMD performance from $\mathrm{C}$ code.

We have also implemented our gravitational interaction functions with both CUDA and OpenCL kernels on NVIDIA GPUs, obtaining single-precision performance of over 2 Tflops on a K20x (Table 3). We have implemented these kernels within $2 \mathrm{HOT}$ and demonstrated a $3 \mathrm{x}$ speedup over using the CPU alone. The ultimate performance of our code on hybrid GPU architectures depends on the ability of the to perform a highly irregular tree-traversal quickly enough to provide the necessary flow of floating-point intensive gravitational interactions. A parallel scan and sort based on our spacefilling curve key assignment is one example of a successful approach 4.

We have generally achieved near $40 \%$ of peak (singleprecision) CPU performance on the supercomputers we have ported our code to over the past 20 years. We are working toward demonstrating the performance of $2 \mathrm{HOT}$ on Titan, using 18,688 NVIDIA K20x GPUs. With 25\% of peak performance, we would obtain near 20 Tflops on that machine.

\subsection{Managing the Simulation Pipeline}

In order to better integrate the various codes involved, and to simplify the management of the multiple configuration files per simulation, we have developed a Python 58] metaprogramming environment to translate a high-level description of a simulation into the specific text configuration files and shell scripts required to execute the entire simulation pipeline. Without this environment, it would be extremely difficult to guarantee consistency among the various components, or to reproduce earlier simulations after new features have been added to the individual software agents. It also allows us to programatically generate the configuration of thousands of simulations at once, that would previously have to be configured manually.

\subsubsection{Task Management}

Modern simulation pipelines present a complex task for queueing systems. Given the flexibility of $2 \mathrm{HOT}$, which can run on an arbitrary number of processors, or be interrupted with enough notice to write a checkpoint, we would like to control our tasks using higher-level concepts. We wish to specify the general constraints on a simulation task and have the system perform it in an efficient manner with as little human attention as possible. For example, "Please run our simulation that will require 1 million core-hours using as many jobs in sequence as necessary on at least 10,000 cores at a time, but use up to $2 \mathrm{x}$ as many cores if the wait for them to become available does not increase the overall wallclock time, and allow our job to be pre-empted by higher-priority jobs by sending a signal at least 600 seconds in advance." Optimal scheduling of such requests from hundreds of users on a machine with hundreds of thousands of processors is NP-hard, but there seems to be ample room for improvement over the current systems, even without an "optimal" solution.

Data analysis often requires many smaller tasks, which queueing systems and MPI libraries have limited support for as well. We have developed an additional Python tool called stask. It allows us to maintain a queue inside a larger PBS or Moab allocation which can perform multiple smaller simulations or data analysis tasks. It has also proven useful to manage tens of thousands of independent tasks for MapReduce style jobs on HPC hardware. For instance, we have used this approach to generate 6-dimensional grids of cosmological power spectra, as well as perform MarkovChain Monte Carlo analyses.

\subsubsection{Checkpoints and I/O}

$2 \mathrm{HOT}$ reads and writes single files using collective MPI/IO routines. We use our own self-describing file format (SDF), which consists of ASCII metadata describing raw binary particle data structures. I/O requirements are driven primarily by the frequency of checkpoints, which is in turn set by the probability of failure during a run. For the production simulations described here, we experience a hardware failure which ends the job about every million CPU hours ( 80 wallclock hours on 12288 CPUs). Writing a 69 billion particle file takes about 6 minutes, so checkpointing every 4 hours with an expected failure every 80 hours costs 2 hours in I/O and saves 4-8 hours of re-computation from the last permanently saved snapshot. At LANL, we typically obtain 5-10 Gbytes/sec on a Panasas filesystem. We have demonstrated the ability to read and write in excess of 20 Gbytes/sec across 160 Lustre OSTs on the filesystem at ORNL. By modifying our internal I/O abstraction to use MPI/IO across 4 separate files to bypass the Lustre OST limits, we have obtained I/O rates of 45 Gbytes/sec across 512 OSTs. These rates are sufficient to support simulations at the $10^{12}$ particle scale at ORNL, assuming the failure rate is not excessive.

\subsubsection{Version Control of Source Code and Data}

To assure strict reproducibility of the code and scripts used for any simulation and to better manage development distributed among multiple supercomputer centers, we use the git version control system [56 for all of the codes in the simulation pipeline, as well as our Python configuration system. We additionally automatically propagate the git tags into the metadata included in the headers of the data which is produced from the tagged software.

\subsubsection{Generating Initial Conditions}

We use the Boltzmann code CLASS [30, 7] to calculate the power spectrum of density fluctuations for a particular cosmological model. A particular realization of this power spectrum is constructed using a version of 2LPTIC [12] we 
have modified to support more than $2^{31}$ particles and use the FFTW3 library.

\subsubsection{Data Analysis}

One of the most important analysis tasks is generating halo catalogs from the particle data by identifying and labeling groups of particles. We use vfind [0] implemented with the HOT library to perform both friend-of-friends (FOF) and isodensity halo finding. More recently, we have adopted the ROCKSTAR halo finder [6], contributing some scalability enhancements to that software, as well as interfacing it with SDF. Our plans for future data analysis involve developing interfaces to the widely-adopted yt Project [57], as well as contributing the parallel domain decomposition and tree traversal technology described here to yt.

Many of the mathematical routines we developed over the years as needed for our evolution or analysis codes have been replaced with superior implementations. The GSL [20] and FFTW 17] libraries have been particularly useful.

\section{SCALABILITY AND PERFORMANCE}

In Table 1 we show the performance of our N-body code on a sample of the major supercomputer architectures of the past two decades. It is perhaps interesting to note that now a single core has more memory and floating-point performance than the fastest computer in the world in 1992 (the Intel Delta, on which we won our first Gordon Bell prize 66]). We show a typical breakdown among different phases of our code in Table 2 , and single processor performance in Table 3.

\begin{tabular}{|c|c|c|r|r|}
\hline Year & Site & Machine & Procs & Tflop/s \\
\hline \hline 2012 & OLCF & Cray XT5 (Jaguar) & 262144 & 1790 \\
\hline 2012 & LANL & Appro (Mustang) & 24576 & 163 \\
\hline 2011 & LANL & SGI XE1300 & 4096 & 41.7 \\
\hline 2006 & LANL & Linux Networx & 448 & 1.88 \\
\hline 2003 & LANL & HP/Compaq (QB) & 3600 & 2.79 \\
\hline 2002 & NERSC & IBM SP-3(375/W) & 256 & 0.058 \\
\hline 1996 & Sandia & Intel (ASCI Red) & 6800 & 0.465 \\
\hline 1995 & JPL & Cray T3D & 256 & 0.008 \\
\hline 1995 & LANL & TMC CM-5 & 512 & 0.014 \\
\hline 1993 & Caltech & Intel Delta & 512 & 0.010 \\
\hline
\end{tabular}

Table 1: Performance of HOT on a variety of parallel supercomputers spanning 20 years of time and five decades of performance.

We present strong scaling results measured on Jaguar in Figure 5. These benchmarks represent a single timestep, but are representative of all aspects of a production simulation, including domain decomposition, tree construction, tree traversal, force calculation and time integration, but do not include I/O (our development allocation was not sufficient to perform this set of benchmarks if they had included $\mathrm{I} / \mathrm{O})$. Also, note that these results were using the code prior to the implementation of background subtraction, so the error tolerance was set to a value resulting in about 4 times as many interactions as the current version of the code would require for this system.

\section{ERROR ANALYSIS}

Verifying the correctness of a large simulation is a complex and difficult process. Analogous to the "distance ladder" in

\begin{tabular}{rr}
\hline computation stage & time $(\mathrm{sec})$ \\
\hline Domain Decomposition & 12 \\
Tree Build & 24 \\
Tree Traversal & 212 \\
Data Communication During Traversal & 26 \\
Force Evaluation & 350 \\
Load Imbalance & 80 \\
\hline Total (56.8 Tflops) & 704 \\
\hline
\end{tabular}

Table 2: Breakdown of computation stages in a single timestep from a recent $4096^{3}$ particle simulation using 2HOT on 12288 processors of Mustang at LANL. The force evaluation consisted of $1.05 \mathrm{e} 15$ hexadecapole interactions, $1.46 \mathrm{e} 15$ quadrupole interactions and $4.68 \mathrm{e} 14$ monopole interactions, for a total of 582,000 floating point operations per particle. Reducing the accuracy parameter to a value consistent with other methods would reduce the operation count by more than a factor of three.

\begin{tabular}{|l|r|}
\hline Processor & Gflop/s \\
\hline \hline 2530-MHz Intel P4 (icc) & 1.17 \\
\hline 2530-MHz Intel P4 (SSE) & 6.51 \\
\hline 2600-MHz AMD Opteron 8435 & 13.88 \\
\hline 2660-MHz Intel Xeon E5430 & 16.34 \\
\hline 2100-MHz AMD Opteron 6172 (Hopper) & 14.25 \\
\hline PowerXCell 8i (single SPE) & 16.36 \\
\hline 2200-MHz AMD Opteron 6274 (Jaguar) & 16.97 \\
\hline 2600-MHz Intel Xeon E5-2670 (AVX) & 28.41 \\
\hline 1300-MHz NVIDIA M2090 GPU (16 SMs) & 1097.00 \\
\hline 732-MHz NVIDIA K20X GPU (15 SMs) & 2243.00 \\
\hline
\end{tabular}

Table 3: Single core/GPU performance in Gflop/s obtained with our gravitational micro-kernel benchmark for the monopole interaction. All numbers are for single-precision calculations, calculated using 28 flops per interaction.

astronomy, where no single technique can measure the distances at all scales encountered in cosmology, we must use a variety of methods to check the results of our calculations. As an example, using the straightforward Ewald summation method to calculate the force on a single particle in a $4096^{3}$ simulation requires over $10^{14}$ floating point operations (potentially using 128-bit quadruple precision), so it is impractical to use for more than a very small sample of particles. However, it can be used to verify a faster method, and the faster method can be used to check the accuracy of the forces in a much larger system. Eventually, we reach the stage where we can use $2 \mathrm{HOT}$ itself to check lower-accuracy results by adjusting the accuracy parameter within the code (as long as we are willing to pay the extra cost in computer time for higher accuracy).

Additionally, writing simple tests to verify the behavior of individual functions is essential. We have used Cython 5] to wrap the functions in $2 \mathrm{HOT}$, allowing them to be tested from within a more flexible and efficient Python environment. In Figure 6 we show one such example, showing the expected behavior of various orders of multipole interactions vs distance.

We also can compare the results of $2 \mathrm{HOT}$ with other codes, and investigate the convergence properties of various 


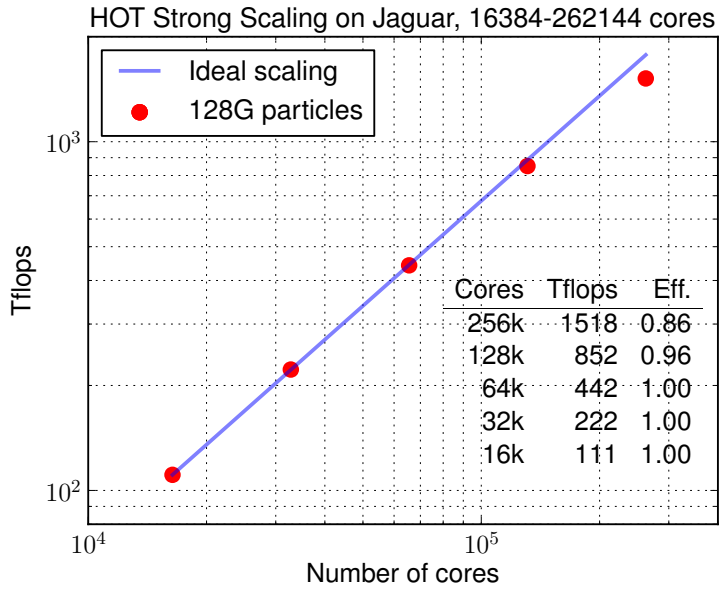

Figure 5: Scaling on Jaguar measured in June 2012.
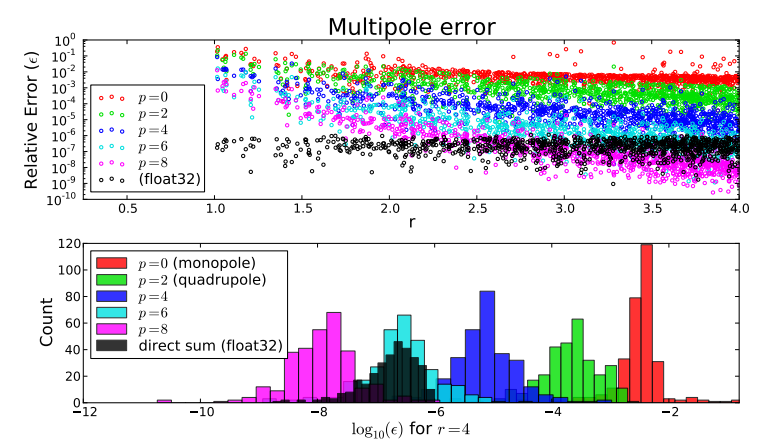

Figure 6: Error behavior for multipoles of various order $(p)$ for 512 particles randomly distributed in a cube of size 1 at distance $r$. A single $p=8$ multipole is more accurate than direct summation in single precision at $r=4$.

parameters. One must always keep in mind that convergence testing is necessary, but not sufficient, to prove correctness. In a complex system there may be hidden parameters that are not controlled for, or variables that interact in an unexpected way, reducing the value of such tests. Having two methods agree also does not prove that they are correct, only that they are consistent.

In Figure 7 we show the sensitivity of the power spectrum to adjustments in various code parameters, as well as comparing with the widely used GADGET2 [50] code. The power spectrum is a sensitive diagnostic of errors at all spatial scales, and can detect deficiencies in both the time integration and force accuracy. We can conclude from these graphs that $2 \mathrm{HOT}$ with the settings used for our scientific results (an error tolerance of $10^{-5}$ ) produces power spectra accurate to 1 part in 1000 at intermediate and large scales, with parameters such as the smoothing length and starting redshift dominating over the force errors at small scales. $2 \mathrm{HOT}$ also systematically differs from GADGET2 at scales corresponding to the switch between tree and particle-mesh, an effect also observed when comparing GADGET2 with perturbation theory results at high redshift 52 .

\section{SCIENTIFIC RESULTS}

The number of objects in the Universe of a given mass is a fundamental statistic called the mass function. The mass function is sensitive to cosmological parameters such as the matter density, $\Omega_{m}$, the initial power spectrum of density fluctuations, and the dark energy equation of state. Especially for very massive clusters (above $10^{15}$ solar masses $\left.\left[M_{\odot} / h\right]\right)$ the mass function is a sensitive probe of cosmology. For these reasons, the mass function is a major target of current observational programs 10. Precisely modeling the mass function at these scales is an enormous challenge for numerical simulations, since both statistical and systematic errors conspire to prevent the emergence of an accurate theoretical model (see 43 and references therein). The dynamic range in mass and convergence tests necessary to model systematic errors require multiple simulations at different resolutions, since even a $10^{12}$ particle simulation does not have sufficient statistical power by itself.

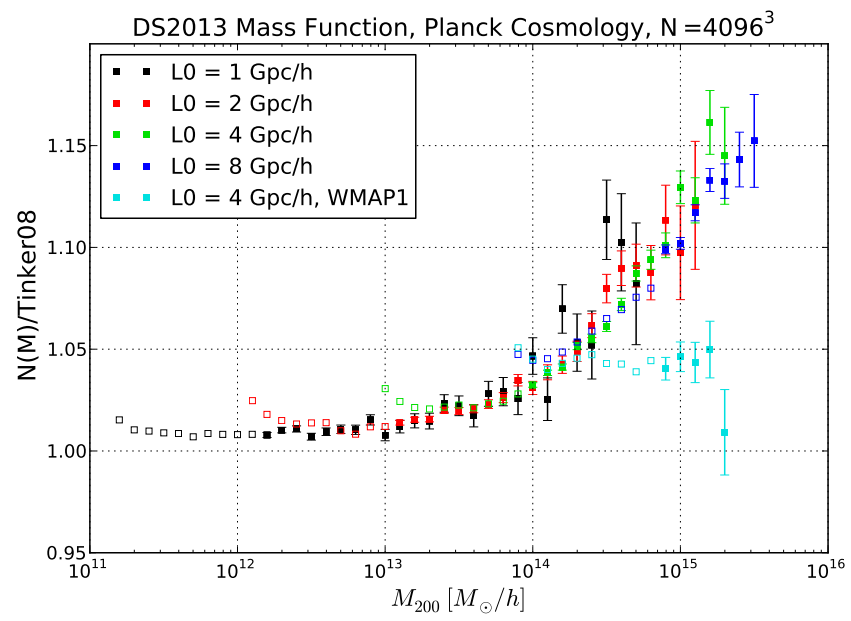

Figure 8: A plot of the mass function from four recent $4096^{3}$ particle simulations computed with 2HOT. The scale of the computational volume changes by a factor of two between each simulation (so the particle mass changes by factors of 8 ). We plot our data divided by the fit of Tinker08 [55] on a linear $y$-axis. The figure shows the simulations are internally consistent but deviate from the Tinker08 fit at large scales. Open symbols are used for halos with 100-1000 particles, showing consistency at the $1 \%$ level down to 200 particles per halo.

Our HOT code was an instrumental part of the first calculations to constrain the mass function at the $10 \%$ level 61] with a series of sixteen $1024^{3}$ simulations performed in 2005 , accounting for about $4 \times 10^{18}$ floating point operations. These results were further refined to a $5 \%$ level of accuracy with the addition of simulations from other codes, and the use of a more observationally relevant spherical overdensity (SO) mass definition [55. With our suite of simulations (twelve $4096^{3}$ simulations, with an aggregate volume of thousands of cubic Gpc, using roughly 20 million corehours and accounting for $4 \times 10^{20}$ floating point operations), we are able to probe effects at the $1 \%$ level in the SO mass function above $10^{15} M_{\odot} / h$ for the first time. 

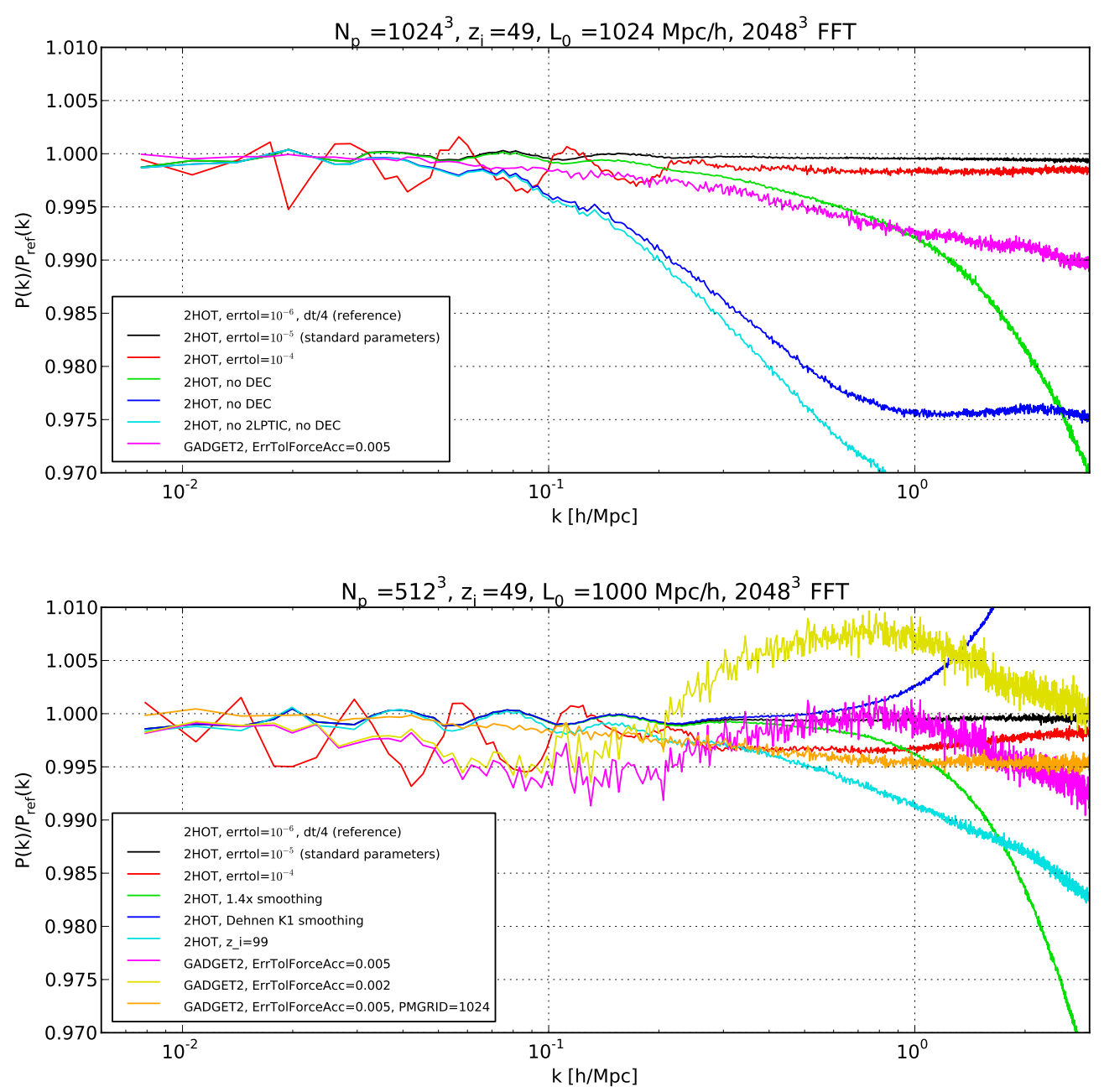

Figure 7: We show the difference between the power spectra at $\mathrm{z}=0$ using 2 HOT and GADGET2 on $1024^{3}$ particles in a $1 \mathrm{Gpc} / \mathrm{h}$ box, as well as variations due to using the 2LPT correction to the initial conditions and discretization error correction (DEC) of the same form as a cloud-in-cell deconvolution.. GADGET2 and 2HOT agree within $0.1 \%$ on large spatial scales, but GADGET2 is about $1 \%$ lower at $k=1$, in its TreePM transition region. With a relaxed error tolerance of $10^{-4}$ (10x the value used for our scientific results, which results in a $3 x$ reduction in the number of interactions) $2 \mathrm{HOT}$ demonstrates errors at the $0.5 \%$ level at large scales, with errors at the $0.1 \%$ level at small scales. Not using 2LPTIC initial conditions reduces the power spectrum at $\mathrm{k}=1$ by more than $2 \%$ (blue curve). The lower panel shows the same graphs using a lower $512^{3}$ particle resolution ( $8 \mathrm{x}$ higher particle mass). We note the GADGET2 results differ among themselves at the $0.5 \%$ level, depending on the chosen parameters. The change in resolution moves the TreePM transition region for GADGET2 to a spatial scale $2 \mathrm{x}$ as large ( $\mathrm{k}$ reduced a factor of 2) compared with the previous figure. The effects of changing the smoothing kernel (blue) and smoothing length (green) are also shown.

Some highlights of our scientific results for the mass function of dark matter halos (Figure 8) are:

- We provide the first mass function calculated from a suite of simulations using the new standard Planck 2013 cosmology (with a $4096^{3}$ particle simulation and six $2048^{3}$ simulations completed and shared with our collaborators within 30 days of the publication of the Planck 2013 results). Changes in the parameters from the previous WMAP7 model are large enough that extrapolations from the other cosmologies [2, 1] are likely subject to systematic errors which are large compared to the statistical precision of our results.

- We find the Tinker08 55 result underestimates the mass function at scales of $10^{15} M_{\odot} / h$ by about $5 \%$ when compared with the older WMAP1 cosmological model it was calibrated against.

- For the Planck 2013 cosmology, the Tinker08 mass function is $10-15 \%$ low at large scales, due to the added systematic effect of non-universality in the underlying theoretical model.

- We identify a systematic error stemming from the im- 
proper growth of modes near the Nyquist frequency, due to the discrete representation of the continuous Fourier modes in the ideal input power spectrum with a fixed number of particles. This is a resolution dependent effect which is most apparent when using particle masses larger than $10^{11} M_{\odot}$ (corresponding to using less than 1 particle per cubic $\mathrm{Mpc} / \mathrm{h}$ ). Uncertainty in the appropriate correction and consequences of this effect appear to be the dominant source of systematic error in our results, where statistical uncertainties prevent us from ruling out a $1 \%$ underestimate of the mass function at scales of $2 \times 10^{15} M_{\odot} / h$ and larger. If uncontrolled, this discretization error confounds convergence tests which attempt to isolate the effects of the starting redshift of the simulation 31, 43, since the error becomes larger at higher starting redshifts.

- We are in direct conflict with recent results 71] (see their Figure 13) which find the SO mass function to be lower than the the Tinker08 result at high masses. Potential explanations would be insufficient force accuracy of the $\mathrm{CUBEP}^{3} \mathrm{M}$ code 24] (c.f. their Figure 7 showing force errors of order $50 \%$ at a separation of a few mesh cells), with a secondary contribution from initial conditions that did not use 2LPT 12 corrections (more recent simulations in 72 appear consistent with our results up to $2 \times 10^{15} M_{\odot} / h$ ).

\section{CONCLUSION}

Using the background subtraction technique described in Section 2.2.1 improved the efficiency of our treecode algorithm for cosmological simulations by about a factor of three when using a relatively strict tolerance $\left(10^{-5}\right)$, resulting in a total absolute force error of about $0.1 \%$ of the typical force. We have evidence that accuracy at this level is required for high-precision scientific results, and we have used that tolerance for the results presented here. That accuracy requires about 600,000 floating point operations per particle (coming mostly from $\sim 2000$ hexadecapole interactions). Relaxing the error parameter by a factor of 10 (reducing the total absolute error by a factor of three) reduces the operation count per particle to 200,000 .

We can compare our computational efficiency with the 2012 Gordon Bell Prize winning TreePM N-body application 26] which used 140,000 floating point operations per particle. The $\theta$ parameter for the Barnes-Hut algorithm in that work was not specified, so it is difficult to estimate the effective force accuracy in their simulation. Modulo being able to precisely compare codes at the same accuracy, this work demonstrates that a pure treecode can be competitive with TreePM codes in large periodic cosmological volumes. The advantage of pure treecodes grows significantly as applications move to higher resolutions in smaller volumes, use simulations with multiple hierarchical resolutions, and require non-periodic boundary conditions.

Our experience with HOT over the past twenty years perhaps provides a reasonable baseline to extrapolate for the next ten years. The Intel Delta machine provided 512 single processor nodes running at $40 \mathrm{MHz}$ and no instruction-level parallelism (concurrency of 512). The benchmark we ran on Jaguar had 16,384 16-core nodes running at $2.2 \mathrm{GHz}$ and 4 wide single-precision multiply-add SSE instructions (concurrency of 2.1 million). The performance difference for HOT of 180,000 between these machines is nicely explained from a factor of 55 in clock rate, a factor of 4096 in concurrency, and the loss of about $20 \%$ in efficiency. (Most of the efficiency loss is simply the fact that the gravitational inner loop can not balance multiplies and adds, so FMA instructions can not be fully utilized).

Looking to the future, if we guess clock rates go down a factor of two for better power utilization, and we lose up to a factor of two in efficiency, we would need an additional factor of 2000 in concurrency to reach an exaflop. A factor of 64 is gained going to 256 -wide vector operations, leaving us with $32 \mathrm{x}$ as many cores. A machine with 8 million cores is daunting, but measured logarithmically the jump from $\log _{2}(512)=9$ on the Delta to $\log _{2}(262144)=18$ on Jaguar is twice as large as the jump from Jaguar to an exaflop machine with $\log _{2}\left(N_{\text {cores }}\right)$ of 23 . Assuming the hardware designers make sufficient progress on power and fault-tolerance challenges, the basic architecture of $2 \mathrm{HOT}$ should continue to serve at the exascale level.

\section{ACKNOWLEDGMENTS}

We gratefully acknowledge John Salmon for his many contributions to the initial version of HOT, and helpful comments on a draft version of this manuscript. We thank Mark Galassi for his memory management improvements to $2 \mathrm{HOT}$ and Ben Bergen for assistance with the OpenCL implementation. We thank the Institutional Computing Program at LANL for providing the computing resources used for our production simulations. This research used resources of the Oak Ridge Leadership Computing Facility at Oak Ridge National Laboratory, which is supported by the Office of Science of the Department of Energy under Contract DE-AC0500OR22725. This research also used resources of the National Energy Research Scientific Computing Center, which is supported by the Office of Science of the U.S. Department of Energy under Contract No. DE-AC02-05CH11231. This research was performed under the auspices of the National Nuclear Security Administration of the U. S. Department of Energy under Contract DE-AC52-06NA25396.

\section{REFERENCES}

[1] R. E. Angulo, V. Springel, S. D. M. White, A. Jenkins, C. M. Baugh, and C. S. Frenk. Scaling relations for galaxy clusters in the Millennium-XXL simulation arXiv:1203.3216, 2012.

[2] R. E. Angulo and S. D. M. White. One simulation to fit them all - changing the background parameters of a cosmological N-body simulation Monthly Notices of the Royal Astronomical Society, 405(1):143-154, 2010.

[3] P. Balaji, et al. MPI on millions of cores Parallel Processing Letters, 21(01):45-60, 2011.

[4] J. Bédorf, E. Gaburov, and S. Portegies Zwart. A sparse octree gravitational N-body code that runs entirely on the GPU processor Journal of Computational Physics, 231(7):2825-2839, 2012.

[5] S. Behnel, R. Bradshaw, C. Citro, L. Dalcin, D. Seljebotn, and K. Smith. Cython: The best of both worlds Computing in Science Engineering, 13(2):31-39, 2011.

[6] P. S. Behroozi, R. H. Wechsler, and H. Wu. The ROCKSTAR phase-space temporal halo finder and the velocity offsets of cluster cores. The Astrophysical Journal, 762(2):109, 2013. 
[7] D. Blas, J. Lesgourgues, and T. Tram. The cosmic linear anisotropy solving system (CLASS). part II: approximation schemes Journal of Cosmology and Astroparticle Physics, 2011(07):034, 2011.

[8] M. Challacombe, C. White, and M. Head-Gordon. Periodic boundary conditions and the fast multipole method The Journal of Chemical Physics, 107(23):10131-10140, 1997.

[9] Planck Collaboration. Planck 2013 results. XVI. cosmological parameters, arXiv:1303.5076, 2013.

[10] Planck Collaboration. Planck 2013 results. XX. cosmology from Sunyaev-Zeldovich cluster counts. arXiv:1303.5080, 2013.

[11] National Research Council. New worlds, new horizons in astronomy and astrophysics National Academies Press, 2010.

[12] M. Crocce, S. Pueblas, and R. Scoccimarro. Transients from initial conditions in cosmological simulations Monthly Notices of the Royal Astronomical Society, 373(1):369-381, 2006.

[13] W. Dehnen. Towards optimal softening in threedimensional N-body codes - I. minimizing the force error. Monthly Notices of the Royal Astronomical Society, 324(2):273-291, 2001.

[14] G. Efstathiou, M. Davis, S. D. M. White, and C. S. Frenk. Numerical techniques for large cosmological Nbody simulations The Astrophysical Journal Supplement Series, 57:241-260, 1985.

[15] C. I. Ellinger, P. A. Young, C. L. Fryer, and G. Rockefeller. A case study of small scale structure formation in 3D supernova simulations, arXiv:1206.1834, 2012.

[16] W. Feng. Making a case for efficient supercomputing. Queue, 1(7):54-64, 2003.

[17] M. Frigo and S. G. Johnson. FFTW: an adaptive software architecture for the FFT. In Acoustics, Speech and Signal Processing, 1998. Proceedings of the 1998 IEEE International Conference on, volume 3, page 1381-1384. 1998.

[18] C. L. Fryer, G. Rockefeller, and M. S. Warren. SNSPH: a parallel three-dimensional smoothed particle radiation hydrodynamics code The Astrophysical Journal, 643(1):292, 2006.

[19] C. L. Fryer and M. S. Warren. Modeling Core-Collapse supernovae in three dimensions. The Astrophysical Journal Letters, 574(1):L65, 2002.

[20] M. Galassi, et al. GNU scientific library, Network Theory, 2007.

[21] K. M. Gorski, E. Hivon, A. J. Banday, B. D. Wandelt, F. K. Hansen, M. Reinecke, and M. Bartelmann. HEALPix: a framework for high-resolution discretization and fast analysis of data distributed on the sphere. The Astrophysical Journal, 622(2):759, 2005.

[22] A. G. Gray and A. W. Moore. N-Body problems in statistical learning. Advances in neural information processing systems, page 521-527, 2001.

[23] M. Griebel and G. Zumbusch. Parallel multigrid in an adaptive PDE solver based on hashing and space-filling curves Parallel Computing, 25(7):827-843, 1999.

[24] J. Harnois-Deraps, U. Pen, I. T. Iliev, H. Merz, J. D. Emberson, and V. Desjacques. High performance P3M N-body code: CUBEP3M. arXiv:1208.5098, 2012.

[25] L. Hernquist, F. R. Bouchet, and Y. Suto. Application of the ewald method to cosmological N-body simula- tions. The Astrophysical Journal Supplement Series, 75:231-240, 1991.

[26] T. Ishiyama, K. Nitadori, and J. Makino. 4.45 pflops astrophysical N-Body simulation on K computer - the gravitational Trillion-Body problem. arXiv:1211.4406, 2012.

[27] P. Jetley, F. Gioachin, C. Mendes, L. Kale, and T. Quinn. Massively parallel cosmological simulations with ChaNGa. In IEEE International Symposium on Parallel and Distributed Processing, 2008. IPDPS 2008, pages 1-12. 2008.

[28] A. Kawai and J. Makino. Pseudoparticle multipole method: A simple method to implement a highaccuracy tree code The Astrophysical Journal Letters, 550(2):L143, 2001.

[29] M. Kuhlen, M. Vogelsberger, and R. Angulo. Numerical simulations of the dark universe: State of the art and the next decade arXiv:1209.5745, 2012.

[30] J. Lesgourgues. The cosmic linear anisotropy solving system (CLASS) I: Overview, arXiv:1104.2932, 2011.

[31] Z. Lukić, K. Heitmann, S. Habib, S. Bashinsky, and P. M. Ricker. The halo mass function: High-Redshift evolution and universality. The Astrophysical Journal, 671(2):1160, 2007.

[32] P. MacNeice, K. M. Olson, C. Mobarry, R. de Fainchtein, and C. Packer. PARAMESH: a parallel adaptive mesh refinement community toolkit. Computer physics communications, 126(3):330-354, 2000.

[33] P. M. Mcllroy, K. Bostic, and M. D. Mcllroy. Engineering radix sort Computing systems, 6(1):5-27, 1993.

[34] J. Mellor-Crummey, D. Whalley, and K. Kennedy. Improving memory hierarchy performance for irregular applications. In Proceedings of the 13th international conference on Supercomputing, ICS '99, page 425-433. ACM, New York, NY, USA, 1999.

[35] M. Metchnik. A Fast N-Body Scheme for Computational Cosmology. Ph.D. thesis, U. Arizona., 2009.

[36] B. Nijboer and F. De Wette. On the calculation of lattice sums Physica, 23(1-5):309-321, 1957.

[37] J. Ousterhout. Why threads are a bad idea (for most purposes). In Presentation given at the 1996 Usenix Annual Technical Conference, volume 5. 1996.

[38] M. Parashar and J. Browne. On partitioning dynamic adaptive grid hierarchies. In System Sciences, 1996., Proceedings of the Twenty-Ninth Hawaii International Conference on ,, volume 1, pages 604-613 vol.1. 1996.

[39] P. J. E. Peebles. Large-Scale Structure of the Universe. Princeton University Press, 1980.

[40] D. W. Pfitzner, J. K. Salmon, T. Sterling, P. Stolorz, and R. Musick. Halo world: Tools for parallel cluster finding in astrophysical N-body simulations In P. Stolorz and R. Musick, editors, Scalable High Performance Computing for Knowledge Discovery and Data Mining, pages 81-100. Springer US, 1998.

[41] P. Ploumhans, G. Winckelmans, J. Salmon, A. Leonard, and M. Warren. Vortex methods for direct numerical simulation of Three-Dimensional bluff body flows: Application to the sphere at re $=300,500$, and 1000. Journal of Computational Physics, 178(2):427-463, 2002.

[42] T. Quinn, N. Katz, J. Stadel, and G. Lake. Time stepping N-body simulations, arXiv:astro-ph/9710043, 1997. 
[43] D. S. Reed, R. E. Smith, D. Potter, A. Schneider, J. Stadel, and B. Moore. Toward an accurate mass function for precision cosmology arXiv:1206.5302, 2012.

[44] A. G. Riess, et al. Type ia supernova discoveries at z $>1$ from the hubble space telescope: Evidence for past deceleration and constraints on dark energy evolution. The Astrophysical Journal, 607(2):665, 2004.

[45] J. K. Salmon and M. S. Warren. Skeletons from the treecode closet, Journal of Computational Physics, 111(1):136-155, 1994.

[46] Z. F. Seidov and P. I. Skvirsky. Gravitational potential and energy of homogeneous rectangular parallelepiped. arXiv:astro-ph/0002496, 2000.

[47] G. F. Smoot, et al. Structure in the COBE differential microwave radiometer first-year maps. The Astrophysical Journal, 396:L1-L5, 1992.

[48] E. Solomonik and L. Kale. Highly scalable parallel sorting. In 2010 IEEE International Symposium on Parallel Distributed Processing (IPDPS), pages 1-12. 2010.

[49] D. N. Spergel, et al. First-year wilkinson microwave anisotropy probe (WMAP) observations: determination of cosmological parameters. The Astrophysical Journal Supplement Series, 148(1):175, 2003.

[50] V. Springel. The cosmological simulation code gadget2. Monthly Notices of the Royal Astronomical Society, 364(4):1105-1134, 2005.

[51] R. M. Stallman. Using and porting the gnu compiler collection Free Software Foundation, 1989.

[52] A. Taruya, F. Bernardeau, T. Nishimichi, and S. Codis. RegPT: direct and fast calculation of regularized cosmological power spectrum at two-loop order. arXiv:1208.1191, 2012.

[53] M. Tegmark, et al. Cosmological parameters from SDSS and WMAP, Physical Review D, 69(10):103501, 2004.

[54] R. Thakur, et al. MPI at exascale Procceedings of SciDAC, 2010.

[55] J. Tinker, A. V. Kravtsov, A. Klypin, K. Abazajian, M. Warren, G. Yepes, S. Gottlöber, and D. E. Holz. Toward a halo mass function for precision cosmology: The limits of universality. The Astrophysical Journal, 688(2):709, 2008.

[56] L. Torvalds and J. Hamano. GIT-fast version control system 2005.

[57] M. J. Turk, B. D. Smith, J. S. Oishi, S. Skory, S. W. Skillman, T. Abel, and M. L. Norman. yt: A multi-code analysis toolkit for astrophysical simulation data The Astrophysical Journal Supplement Series, 192:9, 2011.

[58] G. Van Rossum and F. L. Drake Jr. Python reference manual. Centrum voor Wiskunde en Informatica, 1995.

[59] J. Waldvogel. The newtonian potential of a homogeneous cube. Zeitschrift für angewandte Mathematik und Physik ZAMP, 27(6):867-871, 1976.

[60] M. Warren, J. Salmon, D. Becker, M. Goda, T. Sterling, and W. Winckelmans. Pentium pro inside: I. a treecode at 430 gigaflops on ASCI red, II. Price/Performance of $\$ 50 / \mathrm{mflop}$ on Loki and Hyglac In Supercomputing, ACM/IEEE 1997 Conference, pages 61-61. 1997.

[61] M. S. Warren, K. Abazajian, D. E. Holz, and L. Teodoro. Precision determination of the mass function of dark matter halos The Astrophysical Journal, 646(2):881, 2006.

[62] M. S. Warren, D. J. Becker, M. P. Goda, J. K. Salmon, and T. Sterling. Parallel supercomputing with com- modity components In Proceedings of the International Conference on Parallel and Distributed Processing Techniques and Applications (PDPTA'97), page 1372-1381. 1997.

[63] M. S. Warren and B. Bergen. Poster: The hashed OctTree N-Body algorithm at a petaflop In High Performance Computing, Networking, Storage and Analysis (SCC), 2012 SC Companion:, page 1442-1442. 2012.

[64] M. S. Warren, C. L. Fryer, and M. P. Goda. The space simulator: Modeling the universe from supernovae to cosmology In Proceedings of the 2003 ACM/IEEE conference on Supercomputing, SC '03, page 30-. ACM, New York, NY, USA, 2003.

[65] M. S. Warren, T. C. Germann, P. S. Lomdahl, D. M. Beazley, and J. K. Salmon. Avalon: an Alpha/Linux cluster achieves 10 gflops for $\$ 150 \mathrm{k}$. In Proceedings of the 1998 ACM/IEEE conference on Supercomputing, Supercomputing '98, page 1-11. IEEE Computer Society, Washington, DC, USA, 1998.

[66] M. S. Warren and J. K. Salmon. Astrophysical N-body simulations using hierarchical tree data structures. In Supercomputing '92. Proceedings, page 570-576. 1992.

[67] M. S. Warren and J. K. Salmon. A parallel hashed Oct-Tree N-body algorithm In Proceedings of the 1993 ACM/IEEE conference on Supercomputing, Supercomputing '93, page 12-21. ACM, New York, NY, USA, 1993.

[68] M. S. Warren and J. K. Salmon. A portable parallel particle program Computer Physics Communications, 87(1-2):266-290, 1995.

[69] M. S. Warren, E. H. Weigle, and W. Feng. High-density computing: a 240-processor beowulf in one cubic meter In Supercomputing, ACM/IEEE 2002 Conference, page 61-61. 2002.

[70] M. S. Warren, W. Zurek, P. Quinn, and J. Salmon. The shape of the invisible halo: N-body simulations on parallel supercomputers. AIP Conference Proceedings, 222:216, 1991.

[71] W. A. Watson, I. T. Iliev, A. D'Aloisio, A. Knebe, P. R. Shapiro, and G. Yepes. The halo mass function through the cosmic ages, arXiv:1212.0095, 2012.

[72] W. A. Watson, I. T. Iliev, J. M. Diego, S. Gottlöber, A. Knebe, E. Martínez-González, and G. Yepes. Statistics of extreme objects in the juropa hubble volume simulation arXiv e-print 1305.1976, 2013.

[73] J. J. Willcock, T. Hoefler, N. G. Edmonds, and A. Lumsdaine. $\mathrm{AM}++$ : a generalized active message framework. In Proceedings of the 19th international conference on Parallel architectures and compilation techniques, PACT '10, page 401-410. 2010.

[74] S. Wolfram. The MATHEMATICA ® Book, Version 4. Cambridge University Press, 4 edition, 1999.

[75] J. Wu, Z. Lan, X. Xiong, N. Y. Gnedin, and A. V. Kravtsov. Hierarchical task mapping of cell-based AMR cosmology simulations In $S C$ '12, page 75:1-75:10. IEEE Computer Society Press, Los Alamitos, CA, USA, 2012.

[76] L. Ying, G. Biros, and D. Zorin. A kernelindependent adaptive fast multipole algorithm in two and three dimensions. Journal of Computational Physics, 196(2):591-626, 2004.

[77] R. Yokota. An FMM based on dual tree traversal for many-core architectures arXiv:1209.3516, 2012. 